\title{
Psychische Gesundheit im Alter: Lasst uns mehr dafür tun!
}

Gabriela Stoppe

Interessenverbindungen: Die Autorin schreibt in ihrer Funktion als Leiterin der Arbeitsgruppe Mental Health und alternde Bevölkerung von Swiss Public Health sowie als Vorsitzende der Sektion Old Age Psychiatry der World Psychiatric Association. Es bestehen keine Interessenkonflikte.

\section{Korrespondenz:}

Prof. Dr. med. Gabriela Stoppe Universitäre Psychiatrische Kliniken

Wilhelm Klein-Strasse 27

CH-4012 Basel

Tel. 0613255646

Fax 0613255582

gabriela.stoppe[at]unibas.ch
Die westlichen Gesellschaften altern, und das ist wesentlich ein Ergebnis des medizinischen Fortschritts. Dennoch hat man oft den Eindruck, dass zwar alle alt werden wollen, jedoch keiner alt sein will. Dahinter stehen viele - falsche - Vorstellungen vom Alter. Diese sind besonders bei Ärztinnen und Ärzten wirksam, weil sie eher den kranken Teil der Bevölkerung sehen. In einer Untersuchung an Ärzten und Pflegekräften im Raum Hannover konnte vor wenigen Jahren gezeigt werden, dass Alt und Jung weniger mit dem chronologischen Alter als mit Eigenschaften verbunden wurde. Alt wurde dabei assoziiert mit Langsamkeit, Rigidität, Hilflosigkeit, Abhängigkeit, während Jugend mit Flexibilität, Schnelligkeit, Extroversion und Aktivität verbunden wurde [1].

Natürlich kumulieren mit dem Alter Krankheiten - Stichwort Multimorbidität - und vieles «geht nicht mehr wie früher». Dennoch gelingt es der grossen Mehrheit der älteren Bevölkerung, auch in diesem Lebensabschnitt unabhängig zu leben. Im Alter kommt es zwar einerseits zu einer Reduktion der Informationsverarbeitungsgeschwindigkeit, des Arbeitsgedächtnisses oder auch der kognitiven Flexibilität, jedoch bestehen erhebliche interindividuelle Unterschiede, und diese Funktionen lassen sich trainieren. Emotionale Stabilität und Wissen nehmen mit dem Alter zu. Auch ist die Lebenszufriedenheit im höheren Lebensalter besser als im mittleren [2].

Psychische Erkrankungen sind generell häufig, und das gilt auch für das Alter [3, 4]. Sie betreffen etwa ein Viertel der alten Bevölkerung. Die häufigsten Erkrankungen sind neben den Demenzen die Depressionen, Ängste, Schlafstörungen, Delir und Suchterkrankungen. Sie gehen mit erheblichen Kosten einher, allein für Demenzen in der Schweiz 7 Milliarden Franken (www.alz.ch) [5]. Häufig treten psychische Erkrankungen zusammen mit anderen Bedingungen auf, insbesondere (chronisch) körperlichen Erkrankungen. Wie in allen Altersgruppen beeinflusst psychische ebenso wie somatische Komorbidität sowohl die Prognose als auch die Diagnose und Behandlung beider Erkrankungen negativ. Gleichzeitige Störungen des Hörens, Sehens und der Beweglichkeit beeinträchtigen zudem die Beteiligung am sozialen Leben. Auch Armut, Einsamkeit und negative Einstellung zum Alter und zur psychischen Erkrankung spielen eine wichtige Rolle.

Damit stellt die psychische Erkrankung im höheren Lebensalter ganz andere Anforderungen an die Versorgung als in jüngeren Jahren. Wird eine allein-

\section{Zusammenfassung}

Psychische Erkrankungen betreffen etwa jeden dritten alten Menschen. In der Öffentlichkeit stehen (Alzheimer-)Demenzen im Vordergrund, jedoch sind Krankheiten wie Depression, Angst- und/oder Schlafstörungen oder das Delir nicht nur mindestens so häufig, sondern auch gut behandelbar. Inzwischen gibt es zudem Studien, die sehr wirksame Präventionsmöglichkeiten aufzeigen. Leider fehlt immer noch die nötige Kompetenz in der Versorgung, sowohl für Erkennung als auch Diagnostik und Behandlung. Dies gilt insbesondere für die Psychotherapie, die auch im Alter wirksam ist. Gerade wegen der häufigen körperlichen Komorbidität sind Ärztinnen und vor allem Hausärztinnen in der Schlüsselposition, alten Menschen die richtige Hilfe zu vermitteln. Es würde sich mehr als lohnen, auch wirtschaftlich!

stehende sehbehinderte Frau sich um eine Psychotherapie für ihre Ängste bemühen? Würde eine Patientin in einem Pflegeheim eine Psychotherapie bekommen? Schon über diese Fragen spürt man, wie sehr Versorgungsstrukturen mit Setting und Altersvariablen zu tun haben und wie wenig mit der reinen Indikation. Eine psychische Erkrankung bedeutet auch oft grössere Hilflosigkeit und Abhängigkeit. Diese bilden den Boden für Vernachlässigung, Missbrauch und Gewalt, oft tabuisierte Themen [6].

Die wichtige Frage ist also, wie die nötige Kompetenz zu den Patientinnen kommt. Hierzu ist sicher auch ein Austausch über die Versorgungssektoren hinaus notwendig. So müssen Palliativmedizin und Demenzbetreuung und Suchtmedizin und Altenhilfe miteinander Konzepte entwickeln. Wichtig ist hierfür, dass ausreichend Kompetenz aufseiten der Gesundheitsberufe vorhanden ist, wobei dies sowohl qualitativ als auch quantitativ zu sehen ist. Die Schweiz ist hier insofern vorbildlich, als sie mit den Qualifikationen für Geriatrie ebenso wie für Alterspsychiatrie und -psychotherapie früher als andere Länder dafür gesorgt hat, dass Patientinnen, die für 
sie besonders kompetenten Ärztinnen auch erkennen können. Aber auch hier bleibt sehr viel zu tun.

Alter und psychische Erkrankungen stellen auch zunehmend Krankenhäuser und Pflegeheime vor besondere Probleme. Etwa 12\% der Patientinnen in Krankenhäusern haben eine Demenz. Nicht selten entwickeln diese dort ein Delir. Darüber hinaus gilt es einen besonderen Umgang zu beachten, zum Beispiel was die Berücksichtigung der eventuell gestörten Einwilligungsfähigkeit angeht [7]. Insofern ist das «demenzgerechte Krankenhaus» ein Zukunftsthema. In den Pflegeheimen betreffen Demenzen und auch Depressionen jeweils mehr als 50\% der Bewohnerinnen. Ist das Personal ausreichend ausgebildet? Stehen Behandlungsmöglichkeiten zur Verfügung? Im Folgenden sollen einzelne Krankheiten und Probleme kurz skizziert werden.

\section{Demenzen}

Demenzerkrankungen betreffen mindestens $8 \%$ der über 65-Jährigen und steigen bezüglich der Prävalenz exponentiell mit dem Lebensalter an. Immer noch werden sie in der Regel nicht früh, sondern in der Regel eher spät oder gar nicht diagnostiziert. Nach verschiedenen Untersuchungen werden Demenzkranke medizinisch schlechter versorgt als nicht Demente. Auch existiert eine erhebliche medikamentöse Fehlbehandlung mit Überbehandlung mit sedierenden Psychopharmaka und Unterbehandlung mit Antidementiva [8].

\section{Delir}

Das Delir tritt insbesondere in Krankenhäusern auf und zeigt auch hier eine altersassoziierte Häufigkeitszunahme. Nach Untersuchungen am Basler USB sind über 30\% der älteren Patienten betroffen [9].

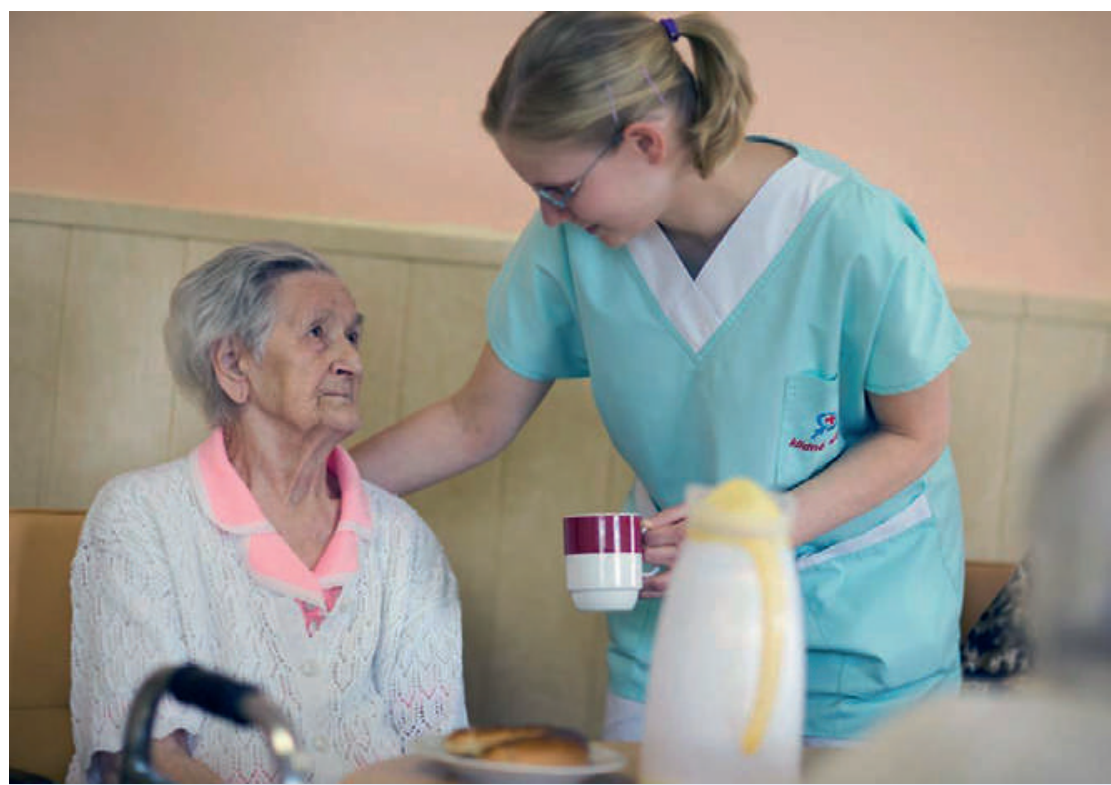

Depressionen, Angststörungen, Demenz, Schlafstörungen - die Beeinträchtigungen für das Wohlbefinden im Alter sind zahlreich.
Vereinfacht gesagt treten Delire auf, wenn der Organismus überfordert ist. Dort, wo man Präventionsprogramme umsetzt, kann die Häufigkeit mindestens halbiert werden. Und dies mit geringem Aufwand (www.delir.info).

\section{Depression}

Dies ist die häufigste psychische Erkrankung auch im Alter. Je nach Schweregrad sind 10-20\% der Bevölkerung von einer Depression betroffen. Viele der Risikofaktoren sind präventiv angehbar, insbesondere andauernde Schlafstörungen, Vereinsamung oder Armut. Die Behandlungsergebnisse und Chancen sind grundsätzlich gleich gut wie bei Jüngeren [10].

\section{Angststörungen}

Diese treten auch im Alter bei 3-14\% auf. Den grössten Anteil machen spezifische Phobien, aber auch generalisierte Angststörungen aus. In der überwiegenden Mehrzahl handelt es sich um altgewordene Patientinnen mit Angsterkrankungen, weil 90\% einen Beginn vor dem 40. Lebensjahr aufweisen. Ein später Beginn kann im Kontext zu körperlichen Erkrankungen stehen. Dies gilt auch bei Depressionen.

Eine besondere Form der Angst im Alter ist die Sturzangst. Interessant ist, dass diese durchaus nicht nur eine Folge von einem Sturz ist, sondern in mindestens gleicher Weise auch ein Risikofaktor für Stürze [11].

\section{Schlafstörungen}

Diese treten mit zunehmendem Lebensalter immer häufiger auf und betreffen bei den über 65-Jährigen fast jede Zweite. Die Mehrheit hat Probleme beim Durchschlafen, gefolgt von Früherwachen und Problemen beim Einschlafen. Neben einer sorgfältigen Schlafanamnese ist auch die Beachtung der Schlafhygiene zu empfehlen. Schlafassoziierte Störungen wie das Schlaf-Apnoe-Syndrom oder auch die vermehrte Bewegungsunruhe bedürfen einer fachgerechten Behandlung [12].

\section{Alkoholabhängigkeit}

Generell werden Abhängigkeitsprobleme im Alter seltener. Bei Untersuchungen in Deutschland zeigte sich bei den über 75-Jährigen eine Häufigkeit von 6,5\% riskantem Trinken (mehr als $20 \mathrm{~g}$ bei Frauen und $30 \mathrm{~g}$ bei Männern). Der schädliche Alkoholkonsum trat bei $0,8 \%$ auf. In Einrichtungen der Altenpflege ist das riskante Trinkverhalten vergleichsweise seltener, jedoch sind gleichzeitig dort Personen mit Folgen schädlichen Gebrauchs häufiger untergebracht, zum Beispiel mit Korsakov-Syndrom und Alkoholdemenz. Hier sind Männer jeweils mehrfach häufiger betroffen als Frauen [4].

\section{Suizid}

Ein besonderes Problem im höheren Lebensalter stellt die hohe Suizidrate dar. Auch wenn Männer bis etwa 
drei Mal häufiger an einem Suizid versterben als Frauen, ist doch jede zweite Frau mit Suizid alter als 60 Jahre [13]. Kürzlich veröffentlichte das Bundesamt für Statistik Zahlen, in denen den Suiziden zugerechnete Fälle von Tod durch Sterbehilfe beziehungsweise assistierten Suizid herausgerechnet waren. Hierbei zeigt sich ein bedenklicher Trend, dass offensichtlich die «Sterbehilfe» zunehmend eine Methode zum Suizid wird.

Zusammen mit der Arbeitsgruppe «Alternde Bevölkerung» von Swiss Public Health haben wir in den letzten Jahren versucht, Erfahrungen und Ideen $\mathrm{zu}$ einer besseren Suizidprävention im höheren Lebensalter zusammenzustellen. Diese wurden vor kurzem publiziert und sind im Internet $\mathrm{zu}$ finden (www.public-health.ch/logicio/client/publichealth/ file/mental/Positionspapier_Suizidpravention_im_ Alter_D_def.pdf). Entgegen vielen Erwartungen sind Suizide auch im höheren Lebensalter selten $\mathrm{Bi}$ lanzsuizide. Vielmehr hängen sie sehr häufig mit schwierigen Lebenssituationen und nicht beziehungsweise ungenügend behandelten Depressionen zusammen. Im Oktober 2012 werden wir ein «Forum Suizidprävention im Alter» ins Leben rufen. Hier soll das Thema disziplinübergreifend diskutiert und der Öffentlichkeit nähergebracht werden.

Zusammengefasst stellt die alternde Bevölkerung eine Herausforderung bezüglich der Versorgung psychischer Erkrankungen dar. Psychotherapeutische Angebote sollten ausgebaut, Versorgungsstrukturen den speziellen Bedürfnissen älterer Menschen angepasst werden, zumal Modelle existieren, die Akzeptanz und Wirksamkeit zeigen (s.o. und 14, 15). Dies zeigt auch der im Auftrag von Gesundheitsförderung Schweiz und Migros Kulturprozent erstellte Bericht Via - Best practice Gesundheitsförderung im Alter» (www.gesundheitsfoerderung.ch/via). Es tut sich viel in diesem Gebiet, und ich möchte alle ermutigen, mitanzupacken. Schliesslich ist die ältere Bevölkerung in besonderem Masse uns Ärztinnen anempfohlen, weil psychische Probleme in diesem Alter selten ohne körperliche Probleme einhergehen. Und es ist bewiesen: Gute Gefühle im Alter verlängern das Leben [16].

\section{Literatur}

1 Flick U, Walter U, Fischer C, Neuber A, Schwartz FW. Gesundheit als Leitidee? Subjektive Gesundheitsvorstellungen von Ärzten und Pflegekräften. Basel, Bern: Huber; 2004.

2 Baltes PB, Staudinger UM, Lindenberger U. Lifespan psychology: Theory and application to intellectual functioning. Ann Rev Psychology 1999; 50: 471-507.

3 Wittchen HU, Jacobi F, Rehm J, Gustavsson A, Svensson M, Johnsson B, et al. The size and burden of mental disorders and other disorders of the brain in
Europe 2010. Eur Neuropsychopharmacol. 2011;21:655-79.

4 Weyerer S. Nicht nur Demenz: Häufigkeit psychischer Erkrankungen im höheren Lebensalter. In: Stoppe G (Hrsg.). Die Versorgung psychisch kranker alter Menschen. Bestandsaufnahme und Herausforderung für die Versorgungsforschung. Köln: Deutscher Ärzte-Verlag; 2011. S. 9-18.

5 Olesen J, Gustavsson A, Svensson M, Wittchen HU, Johnsson B; CDBE2010 study group; European Brain Council. The economic cost of brain disorders in Europe. Eur J Neurol. 2012;19:155-62.

6 Hirsch RD. Gewalt gegen alte Menschen: handeln statt misshandeln. In: Stoppe G (Hrsg.) Die Versorgung psychisch kranker alter Menschen. Bestandsaufnahme und Herausforderung für die Versorgungsforschung. Köln: Deutscher Ärzte-Verlag; 2011 S. 275-86.

7 Peisah C, Finkel S, Shulman K, Melding P, Luxenberg J, et al. for the International Psychogeriatric Association Task Force on Wills and Undue Influence. The wills of older people: risk factors for undue influence. Int Psychogeriatr. 2009;2:7-15.

8 Waldemar G, Phung KTT, Burns A, Georges J, Hansen FR, Iliffe S, et al. Access to diagnostic evaluation and treatment for dementia in Europe. Int J Geriatr Psychiatry. 2007; 22: 47-54.

9 Hasemann W, Kressig RW, Ermini-Fünfschilling D, Pretto M, Spirig R. Delirien. Pflege 2007;20: 191-204.

10 Stoppe G. Depression im Alter. Bundesgesundheitsbl Gesundheitsforsch Gesundheitsschutz. 2008;51: 406-10.

11 Wolitzky-Taylor KB, Castriotta N, Lenze EJ, Stanley MA, Craske MG. Anxiety disorders in older adults: a comprehensive review. Depress Anxiety. 2010;27: 190-211.

12 Staedt J. Schlafstörungen im Alter: Insomnie, Erkennung, Prävention und Behandlung. In: Stoppe G (Hrsg.) Die Versorgung psychisch kranker alter Menschen. Bestandsaufnahme und Herausforderung für die Versorgungsforschung. Köln: Deutscher Ärzte-Verlag; 2011, S. 49-54.

13 Schaller A, Schmidtke S. Suizidalität: Epidemiologische Befunde, Probleme und Schlussfolgerungen für die Prävention. In: Stoppe G (Hrsg.). Die Versorgung psychisch kranker alter Menschen. Herausforderungen für die Versorgungsforschung. 3. Report Versorgungsforschung. Köln: Deutscher Ärzteverlag; 2011, S. 39-48.

14 Maercker A, Enzler A, Grimm G, Helfenstein, Ehlert U. Inanspruchnahme und Psychotherapiemotivation in einer repräsentativen Bevölkerungsstichprobe über 65?jähriger - Ergebnisse der Zürcher Altersstudie. Psychotherap Psychosom Med Psychol. 2005;55: $177-82$.

15 van't Veer-Tazelaar PJ, van Marwijk HW, van Oppen P, van der Horst HE, Smit F, Cuijpers P, et al. Prevention of late-life anxiety and depression has sustained effects over 24 months: a pragmatic randomized trial. Am J Geriatr Psychiatry. 2011;19:230-9.

16 Steptoe A, Wardle J. Enjoying life and living longer. Arch Intern Med. 2012;172:273-5. 